\title{
DÜBLIN
}

Technological University Dublin ARROW@TU Dublin

2007-01-01

\section{Making OLSR Aware of Resources}

\author{
Karol Kowalik \\ Technological University Dublin, Karol.kowalik@tudublin.ie \\ Brian Keegan \\ Technological University Dublin, brian.x.keegan@tudublin.ie \\ Mark Davis \\ Technological University Dublin, mark.davis@tudublin.ie
}

Follow this and additional works at: https://arrow.tudublin.ie/commcon

Part of the Electrical and Electronics Commons

\section{Recommended Citation}

Kowalik, K., Keegan, B. \& Davis, M. (2007) Making OLSR aware of resources. International Conference on Wireless Communcations, Networking and Mobile Computing, WiCom 2007, pp.1488-1493. 21-25th of September, 2007,

This Conference Paper is brought to you for free and open access by the Communications Network Research Institute at ARROW@TU Dublin. It has been accepted for inclusion in Conference papers by an authorized administrator of ARROW@TU Dublin. For more information, please contact arrow.admin@tudublin.ie, aisling.coyne@tudublin.ie,gerard.connolly@tudublin.ie.

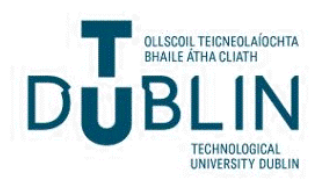




\title{
Making OLSR Aware of Resources
}

\author{
Karol Kowalik, Brian Keegan and Mark Davis \\ Communications Network Research Institute, \\ Dublin Institute of Technology \\ IRELAND \\ Email:karol.kowalik@cnri.dit.ie,brian@cnri.dit.ie,mark.davis@dit.ie
}

\begin{abstract}
An important element of any routing protocol used for Wireless Mesh Networks (WMNs) is the link cost function used to represent the radio link characteristic. The majority of the routing protocols for WMNs attempt to accurately characterise the radio link quality by constructing the link cost function from the measurements obtained using active probing techniques which introduces overhead. In this paper we present a modified version of the Optimized Link State Routing (OLSR) protocol which uses the link cost function values provided the Resource Aware Routing for mEsh (RARE) module which employs passive monitoring to gather radio link information. This results in a smaller overhead than the other methods that require active network probing and furthermore is load independent since it does not require an access to the medium. We demonstrate the necessary modifications to OLSR required to make it work with RARE. The results of our ns-2 simulations show that such a combination (OLSR + RARE) performs well over various wireless topologies.
\end{abstract}

\section{INTRODUCTION}

Wireless Mesh Networks (WMNs) [1] are a type of radiobased network system which require minimal configuration and infrastructure. This technology allows for quick and inexpensive deployment of wireless local area networks (WLANs). The typical wired LAN is configured using static routes. However, the dynamics of the radio environment require the use of routing protocols which can dynamically adapt their routes according to changes in the network environment. WMNs can change in the following ways:

- their link characteristics change - this is because of external sources of interference; moving nodes and obstacles; and interference from other nodes.

- their topology changes - this is because changes in the link characteristic may result in the loss of connectivity, changing the whole topology of the network.

The choice of radio technology for WMNs influences the performance of the network. Consequently, the routing protocol needs to be aware of this and cannot operate in the same way as wired networks which are often agnostic about the underlying communication medium. Researchers have proposed a variety of new routing protocols developed specifically for ad-hoc wireless mesh networks which are often tailored to meet the requirements of the radio or the application for the mesh technology.

Any routing protocol which aims to find stable and high throughput paths for demanding users must be aware of the underlying radio and needs to accurately represent it using an appropriate link cost function. Also because of the dynamics of the wireless network, the nodes need to be capable of tolerating imprecise state information. To deal with the dynamics and hence inaccuracy of the routing information, routing protocols adapt to changes in a proactive (OLSR [2] or HLSL [3]) or reactive (DSR [4] or AODV [5]) manner.

In this paper we focus on one proactive routing protocol - Optimized Link State Routing (OLSR) protocol which allows for the efficient dissemination of link state information. Especially when combined with RARE module which is responsible for mapping of the radio link characteristics into the link cost function, it can fully exploit information about the underlying medium. The RARE module uses passive monitoring of the wireless medium. We show that by using passive monitoring we can create a sufficiently accurate link representation that will allow OLSR to select high throughput and high quality paths. Another important benefit of our approach is that RARE eliminates the overhead associated with probing the network used by such metrics as Expected Transmission Count (ETX) [6], Expected Transmission Time (ETT) [7], or Weighted Cumulative Expected Transmission Time (WCETT) [8].

\section{RARE}

An accurate representation of the quality of the wireless link using a link cost function has a major influence on the performance of the wireless network. The wireless links which exhibit bad quality should not be used. Only symmetric links with high data rate allow for selection of high throughput paths and stable network operation.

The Resource Aware Routing for mEsh (RARE) is a routing module which aims to make a routing protocol aware of the wireless medium. It obtains the information about the wireless resources through passive monitoring. It measures the three elements which have been recognised as significantly influencing the performance of wireless mesh networks namely the signal strength, the contention, and the available bandwidth.

Signal strength - By selecting paths with strong signal we aim to select paths which can support high-data rates with small error rates. However, the correlation between delivery probability and signal strength may depend on the particular receiver used (as demonstrated by the diagrams in [9]). Therefore, researchers often prefer to actively measure the delivery rate of the link instead of its signal strength [6], [8], [10]. In practice such an approach requires each node to broadcast link 
probe packets, calculate how many of these probe packets it has received, and feedback the results of such calculations to other nodes. However, this generates measurement overhead. Our approach is to use the received signal strength indicator (RSSI), which the IEEE 802.11 proposes to report the RF energy level. Even though it does not reflect the delivery probability as well as the actively measured values there is still a good correlation between delivery probability and signal strength [11].

Interference - Interference has been identified as a key cause of performance degradation in WMNs [12], [13]. Thus, researchers have proposed to actively measure it by checking how transmission activity on one link influences the throughput on other links [13]. However, this procedure for a network with $n$ nodes requires the testing of $n^{4}$ pairs of nodes (or $n^{2}$ when a simplified procedure is employed) while other nodes need to remain idle. Such procedure is time consuming and difficult to realise on a live network. Therefore, we propose a simpler method which estimates the average contention instead of interference. Here the average contention is defined as the number of nodes on average competing for each transmission opportunity (as determined by the MAC layer mechanism). To measure this we put the wireless card into the RFMON (Radio Frequency Monitoring) mode and monitor when multiple stations are contending for access and as such we can obtain the average level of contention.

Bandwidth - The amount of bandwidth available for the data transfers is also an important factor. Draves et al. [8] have proposed measuring it using the technique described in [14]. This is based on sending both small and large probe packets whereby the bandwidth is estimated by dividing the size of the large probe by the time difference between the receipt of the small and the large probe packets. Bicket et al. [7] simplified this procedure which reduces the measurement overhead by using broadcast packets instead. We propose to estimate the available bandwidth by passively monitoring the activities of the nodes, the rates used for data transmission, and the packet sizes used.

Thus the methods which we propose for obtaining the signal strength, interference and bandwidth estimates are non intrusive and do not generate a measurement overhead. These methods are based on passive monitoring which allows a wireless node to intercept the transmission activities of other radios within its communication range. The passive monitoring and statistical analysis of available bandwidth, average contention (amongst other metrics) is performed by a WLAN Resource Monitor [15] application developed at our laboratory. The use of all three elements signal strength, interference, and bandwidth diversifies the path selection criteria and instead of optimising just one of the performance metrics, provides a trade-off which potentially can offer fairer access to the medium.

These three elements are used to construct the radio-aware link cost function. RARE also employs extended HELLO messages (as suggested in [16]) to counteract the link asymmetry however we further extend them to include information about the signal strength. This allows RARE to detect how strong the radio signal is in both directions and consequently to determine if the link can be used for data transmissions.

The RARE link cost function involves three parts comprising bandwidth, contention, and signal strength measurements. The form of the link cost function formula is as follows:

Link_Cost $=\alpha \frac{C-B W_{a}}{B W_{a}}+\beta \frac{R S S I_{\max }-R S S I}{R S S I}+\gamma * N_{c}$

where:

$$
\begin{array}{ll}
B W_{a} & \text { is the available bandwidth } \\
C & \text { is the link capacity } \\
R S S I & \text { is the signal strength (RSSI) value } \\
R S S I_{\max } & \text { is the maximum signal strength } \\
& \text { (RSSI) value } \\
N_{c} & \begin{array}{l}
\text { is the average contention } \\
\alpha, \beta, \gamma
\end{array} \\
& \begin{array}{l}
\text { are the weights associated with the } \\
\text { bandwidth, RSSI and contention } \\
\text { components }
\end{array}
\end{array}
$$

In a wireless environment the values of available bandwidth, signal strength, and contention tend to exhibit significant variations. Therefore, instead of using their current values we smooth the data using an exponential weighted moving average filter which reduces the probability of traffic oscillations, improves reliability, and leads to the selection of more stable paths.

The details description of the meaning of the formula can be found in [17].

\section{OLSR MODIFICATIONS}

The Optimized Link State Routing (OLSR) [2] protocol is a link state routing protocol which uses an efficient method of dissemination of the link state information. This is achieved by the use of "multipoint relays" (MPR) which are the only nodes responsible for forwarding control traffic. Thus OLSR can be used to compute high quality wireless path by the use of link cost information which reflects the quality of the wireless links. However, by default OLSR computes only shortest routes in terms of number of hops. Quality of Service for Ad hoc Optimized Link State Routing Protocol (QOLSR) [18] was proposed to alleviate this problem. However, QOLSR uses multi-constrained path computations (which despite of the use of the shortest-widest path algorithm for best effort traffic and heuristic based on Lagrangian relaxation [19]) introduces computational burden on the mesh nodes. We believe that an accurate representation of the quality of a wireless link can be performed using a single link cost function which combines the information about the signal strength, bandwidth and the interference. Moreover, by the use of a single metric the updates for individual links do not need to be triggered as frequently as for multiple metrics. Furthermore, such updates contain less information, thus overhead involved in their dissemination is lower.

In this paper we propose a simplified version of the QOLSR. It uses only a single metric provided by RARE and employs Dijkstra's algorithm for path computations. However, to enable 
path computation based on a metric different than the hop count the standard OLSR methods and messages need to be modified. Namely the following needed to be extended: (i) HELLO messages - need to be extended to carry for each of the neighbouring nodes information about the link costs and the signal strength (the signal strength information is required in our approach to detect symmetrical links). (ii) TC messages - need to be extended to carry for each of the links information about the link cost. (iii) sets such as Link Set, Neighbour Set, 2-hop Neighbour Set and the Topology Information Base, need to be extended to store information about the link costs. (iv) MPR Computation - for the MPR set computation we propose to modify the heuristic proposed in [2]. Thus after the selection of nodes in the direct neighbourhood which are the only nodes to provide reachability to a node located two hops away, we propose to select the other MPRs based on the link cost to fully cover the nodes located two hops away. Such a heuristic will still limit the overhead involved in dissemination of status information, but it will also give priority to links with the smallest cost. (v) Routing table calculation - for the routing table computation we propose to modify the procedure proposed in [2]. We have decided simply to use the Dijksta's algorithm based on the link cost values stored in the Link Set, Neighbour Set, 2-hop Neighbour Set and the Topology Information Base. This way optimal path in terms of smallest cost can be computed.

The OLSR also implements a mechanism for detection of link asymmetry. This is achieved by link sensing. Each node advertises 1-hop neighbourhood which allows other nodes check if the communication between the two nodes is bidirectional and if so then the link can be considered symmetric (for the duration of L_SYM_time). This allows OLSR to select only the wireless links which can be used for data transfers. The link symmetry check is also performed by the RARE which uses the RSSI value computed in both directions to detect whether the link is symmetrical or not. Thus the computation used by RARE extends the simple check performed by OLSR.

\section{IMPLEMENTATION}

For the purpose of evaluation of our approach we have used the ns-2 network simulator. We have modified the UMOLSR [20] source code which is one of the available implementations of the OLSR routing protocol for the ns-2. The UM-OLSR is compliant with RFC 3626 and supports all the functions of OLSR and moreover it implements the linklayer feedback option. All the modifications described in the previous section where applied to UM-OLSR code.

We have also ported the RARE routing module to ns-2. The RARE has been implemented in our previous work using the Click Modular Router Software [21]. In our previous work we have deployed it over the testbed which consists of 17 static nodes based on the Soekris net4521 board. (The results obtained from the testbed can be found in [17]). A part of the RARE routing module is a Wireless Resource Monitor (WRM) [15] which is responsible for the computation of available bandwidth, contention, and other bandwidth components.
To simulate as realistic propagation conditions as possible we have used the realistic channel propagation model by $\mathrm{Wu}$ Xiuchao [22] and the 802.11 bug fixes by Felix SchmidtEisenlohr [23].

\section{REsults}

In this section we compare the overhead and performance of the modified version of OLSR with its default implementation. The modified version uses the RARE link cost function, thus it adapts to changes in available bandwidth, contention and signal strength. The default implementation computes paths with minimal number of hops. In the following section we will refer to these two versions simply by RARE and MINHOP.

The weights for the RARE link cost function were obtained heuristically based on the experimental results and after some preliminary testing and we have specified them as follows: $\alpha=1, \beta=1$, and $\gamma=10$.

In all the simulation scenarios we have used UDP traffic flows with a packet size of 512 bytes. The flows were generated randomly with inter-arrival times and duration times distributed according to Pareto's distribution. We have only modified the packet inter-arrival times to obtain various flow sending rates.

\section{A. Overhead evaluation}

The proposed OLSR modifications alter the MPR set selection process, thus we have expected that the size of the MPR set will increase. The more nodes that broadcast Topology Control (TC) messages the higher overhead associated with the dissemination of such a state information is produced. However, it can be observed in Figure 1(a) that the number of MAC transmissions required by the RARE version of OLSR and by the default MIN-HOP implementation are almost the same.

Figure 1(a) presents the distribution of the OLSR messages and how these messages are combined into single OLSR packets. It can be observed in Figure 1(a) that some HELLO messages are combined with TC messages. However, the most important observation here is that the ratio of the TC messages to other messages generated by OLSR has not increased after OLSR was modified.

Figure 1(a) also reflects the fact that in our simulations there were no MID messages because we have used only nodes with single wireless interfaces.

Figure 1 was generated for a case when the mesh network which was carrying only a small amount of data. However, Figure 2 presents a scenario in which the volume of the traffic increases. Therefore, the ratio of the number of OLSR packets to the number of data packets decreases. Here it can be observed that the overhead associated with the dissemination of the OLSR status information decreases with higher volumes of traffic. Moreover, Figure 2 shows that the overhead of the modified version of OLSR and its default implementations are almost identical for all the traffic volumes. 


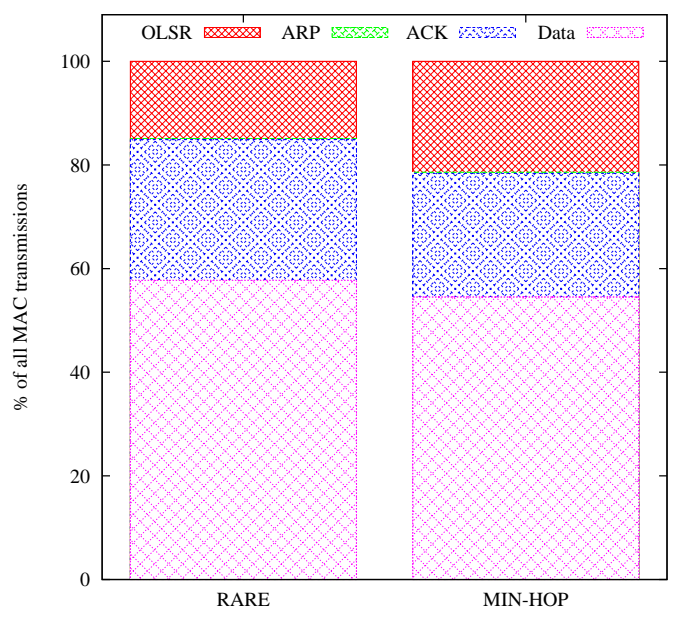

(a) MAC transmission

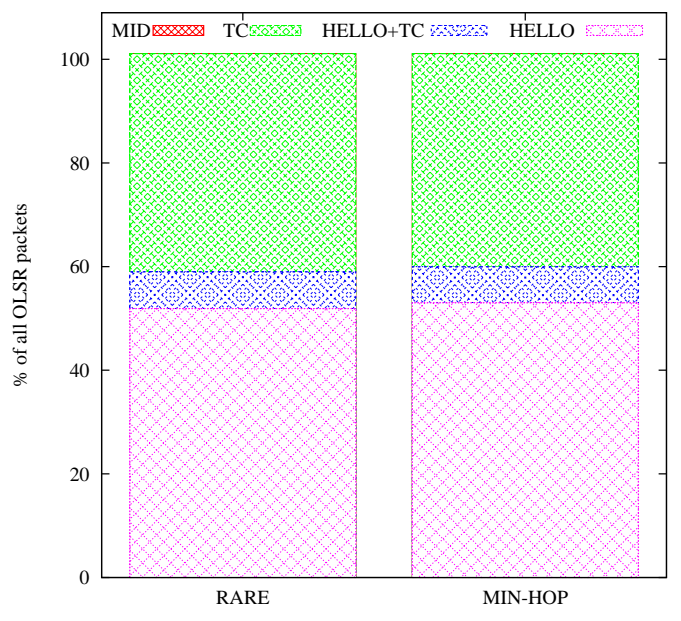

(b) OLSR transmissions

Fig. 1. MAC and OLSR Transmissions

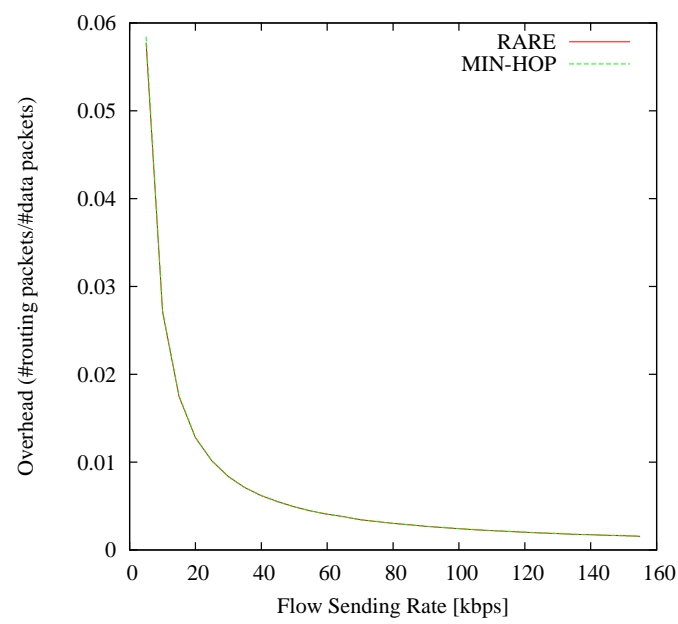

Fig. 2. OLSR overhead

In summary, we have shown that our OLSR modifications have altered the process of dissemination of the status information in a limited way and therefore the RARE version of OLSR does not produce additional overhead.

\section{B. Performance evaluation}

In the first case we study the overall network throughput of RARE and MIN-HOP versions of OLSR when used for a network with topology show in Figure 3(b). In the Figures 3(b) and 4(b) outlining the network topologies we draw a circle around each node to represent the connectivity range of the node.

In this scenario we have specified the traffic pattern in a way that there are 6 source nodes numbered from 0 till 6 . All the sources initiate traffic flows to the destination nodes 10, 11, and 12. The duration of the flows is distributed accordingly to Pareto distribution with the average of 30 seconds, and the inter-flow interval is also distributed accordingly to Pareto distribution with the average of 10 seconds. In this scenario the three source nodes $0 . .2$ can use two alternative paths to the destination. They can use the four-hop path $s \rightarrow 13 \rightarrow$ $14 \rightarrow 15 \rightarrow d$ or the five-hop path $s \rightarrow 6 \rightarrow 7 \rightarrow 8 \rightarrow 9 \rightarrow d$ (where $s$ and $d$ stand for source and destination respectively). The other three source nodes $3 . .5$ can also use two paths, however since the shorter path $s \rightarrow 14 \rightarrow 15 \rightarrow d$ is only three hop long and the other path is eight hops long, thus likely is that they will never use the long path.

The path selection is performed using the OLSR routing protocol. In the case where the default MIN-HOP link cost is used the traffic from the sources aggregates at nodes 14 and 15 , since these two nodes are present in both min-hop paths. Such a traffic distribution works fine for small data rates until the min-hop path saturates. This can be observed at the leftmost side of Figure 3(a). However, after the data rates increase, the min-hop path saturates and consequently the RARE link cost metric forces the OLSR send a portion of the traffic from nodes $0 . .2$ through the longer five hops path $s \rightarrow 6 \rightarrow 7 \rightarrow$ $8 \rightarrow 9 \rightarrow d$. This produces an increase in the overall network throughput and reduction of contention.

In the first example we have demonstrated a case for which RARE was able perform load balancing, and avoid aggregation of traffic along the min-hop path. In the second example, we will present how RARE is able to direct traffic around regions with high contention.

In the second case we study the overall network throughput of RARE and MIN-HOP versions of OLSR when used for a network with topology show in Figure 4(b). We have specified the traffic pattern in a way that the 3 source nodes numbered from 4 till 6 send traffic flows to the destination node 2 . The duration of each flow is distributed accordingly to Pareto distribution with the average of 30 seconds and the inter-flow interval is also distributed accordingly to Pareto distribution with the average of 10 seconds.

In this scenario the three source nodes $4 . .6$ can use two 


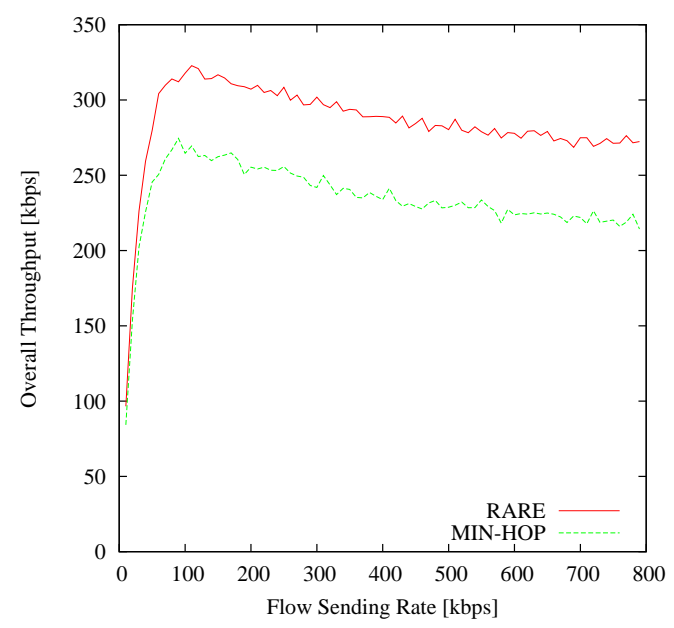

(a) Overall network throughput

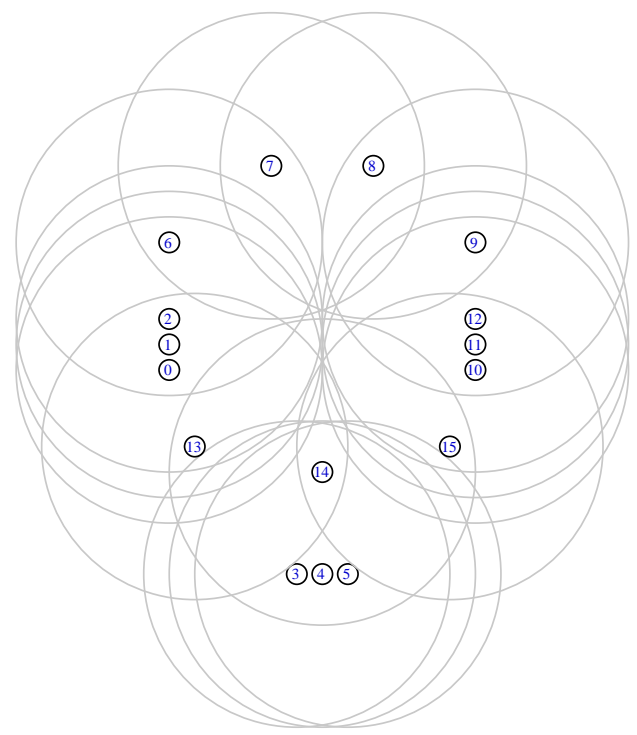

(b) Network topology

Fig. 3. Network throughput and its topology

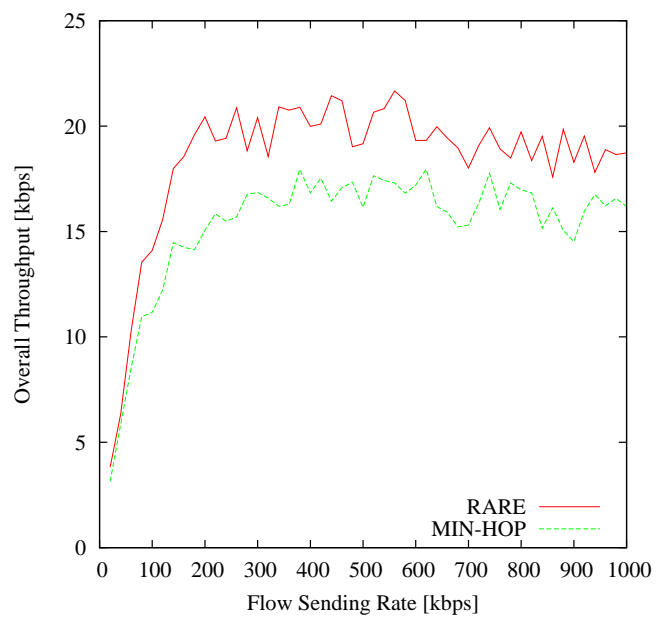

(a) Overall network throughput

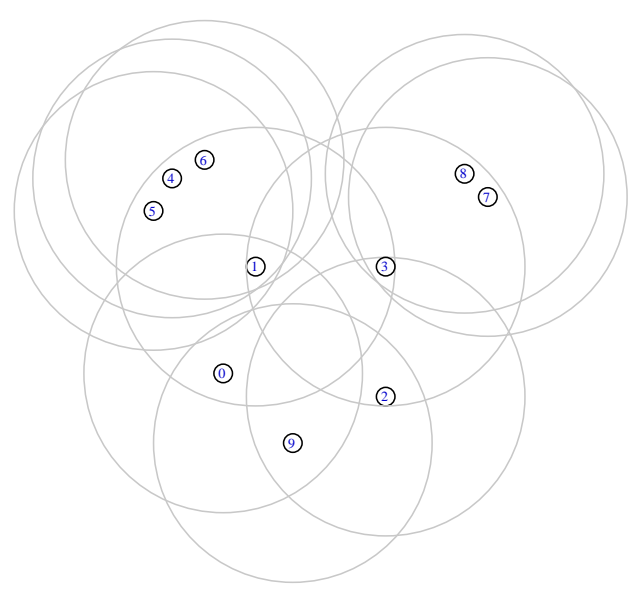

(b) Network topology

Fig. 4. Network throughput and its topology

alternative paths which will lead them to the destination. They can follow a three-hop path $s \rightarrow 1 \rightarrow 3 \rightarrow 2$ or a four-hop path $s \rightarrow 1 \rightarrow 0 \rightarrow 9 \rightarrow 2$ (where $s$ stands for source node, and the destination is always node 2). In this topology we have introduced also an additional traffic flow, between nodes 7 and 8 which is a high throughput data flow of $2 \mathrm{Mbps}$, which causes high contention and reduced throughput for node 3 .

Even though the propagation conditions along the minhop path have deteriorated, the MIN-HOP version of OLSR continues to use it. This results in a lower throughput than RARE which can be observed in Figure 4(a). RARE in turn uses a link cost function that computes link cost value based on signal strength available bandwidth and contention. Therefore, this causes heavily used links to be advertised with a high cost. This in turn directs a portion of the traffic over the path with a higher number of hops but with a smaller cost. Thus RARE again is able to direct the traffic around the region with high contention, while the MIN-HOP continued to use the paths with minimum number of hops.

In summary, our simulation results have shown that the OLSR with RARE approach can perform well in the path selection process, selecting high quality links and balancing the load when the links approach saturation. Moreover, the OLSR modifications do not introduce additional overhead even though they alter the MPR selection mechanism. What is also important is that the RARE module uses passive monitoring technique to obtain the quality of a wireless link and this eliminates the measurement overhead of such a link cost metrics as ETT or ETX.

\section{Conclusions}

In this paper we have introduced a modified version of the OLSR routing protocol. This version extends some of the 
OLSR messages and modifies the MPR and route selection processes. However, these modifications do not produce an additional overhead. Moreover, they onlt alter the mechanism of dissemination of the status information in a minor way, thus the proportion of each of the OLSR messages is not affected by these modifications. The modified version of OLSR uses a novel routing module which we call Resource Aware Routing for mEsh (RARE). This module combines passive measurements of bandwidth, contention, and signal strength in the calculation of the link cost function. This module allows the modified version of OLSR to become more aware of wireless resources and perform load balancing across alternative paths.

Moreover, in our approach we have used the RARE module which is based on passive monitoring (unlike ETT or ETX link cost metrics). It eliminates the need to introduce additional traffic onto the network and is therefore load independent and can provide additional measures regarding the quality of the link without an associated measurements penalty.

We have compared the performance of the modified OLSR version that uses RARE module with that of the default OLSR implementation which is optimised for MIN-HOP computations using the ns-2 network simulator package. Our results show that the modified OLSR and RARE combination performs better than the default OLSR implementation. This is evident when networks approaches saturation conditions or then the contention increases, or the signal strength deteriorates. Then if the network topology features additional paths which could be used for load balancing purposes, RARE is able of finding these paths and in doing so increases the overall network performance.

\section{ACKNOWLEDGEMENT}

The authors acknowledge the support of Enterprise Ireland (under the Commercialisation Fund 2007) and Science Foundation Ireland (under the grant 03/IN3/I396).

\section{REFERENCES}

[1] I. F. Akyildiz and Xudong Wang. A survey on wireless mesh networks. IEEE Communications Magazine, 43(9):23-30, September 2005.

[2] T. Clausen and P. Jacquet. RFC 3561: Optimized Link State Routing Protocol (OLSR), October 2003. Available at http://www. ietf.org/ rfc/rfc3626.txt.

[3] C. Santivanez and R. Ramanathan. Hazy Sighted Link State (HSLS) Routing: A Scalable Link State Algorithm. BBN technical memo BBNTM-1301, BBN technologies, August 2001. Available at http://www . ir.bbn.com/documents/techmemos/index.html.

[4] David B. Johnson, David A. Maltz, and Yih-Chun Hu. The Dynamic Source Routing protocol for mobile ad hoc networks (DSR), July 2004. Internet draft (work in progress), Available at http://www.ietf.org/ internet-drafts/draft-ietf-manet-dsr-10.txt.
[5] C. Perkins, E. Belding-Royer, and S. Das. RFC 3561: Ad hoc OnDemand Distance Vector (AODV) Routing, July 2003. Available at http: //www. ietf.org/rfc/rfc3561.txt.

[6] Douglas S. J. De Couto, Daniel Aguayo, John Bicket, and Robert Morris. A High-Throughput Path Metric for Multi-Hop Wireless Routing. Proceedings of the 9th ACM International Conference on Mobile Computing and Networking (MobiCom '03), September 2003.

[7] John Bicket, Daniel Aguayo, Sanjit Biswas, and Robert Morris. Architecture and Evaluation of an Unplanned 802.11b Mesh Network. MobiCom, August 2005.

[8] R. Draves, J. Padhye, and B. Zill. Routing in Multi-radio, Multi-hop Wireless Mesh Networks. ACM MobiCom, September 2004. Available at http://research.microsoft.com/mesh/papers/multiradio. pdf.

[9] Daniel Aguayo, John Bicket, Sanjit Biswas, Glenn Judd, and Robert Morris. Link-level Measurements from an 802.11b Mesh Network. SIGCOMM 2004, August 2004. Available at http://pdos.lcs.mit. edu/ rtm/papers/p442-aguayo.pdf.

[10] Gautam Kulkarni, Alok Nandan, Mario Gerla, and Mani Srivastava. A Radio Aware Routing protocol for Mesh Networks. Submitted to IEEE Globecom, 2005. Available at http://www.cs.ucla.edu/ alok/ papers/radio.pdf.

[11] Charles Reis, Ratul Mahajan, Maya Rodrig, David Wetherall, and John Zahorjan. Measurement-Based Models of Delivery and Interference in Static Wireless Networks. ACM SIGCOMM, September 2006.

[12] K. Jain, J. Padhye, V. N. Padmanabhan, , and L. Qiu. Impact of interference on multi-hop wireless network performance. ACM MobiCom, September 2003.

[13] Jitu Padhye, Sharad Agarwal, Venkat Padmanabhan, Lili Qiu, Ananth Rao, and Brian Zill. Estimation of Link Interference in Static Multi-hop Wireless Networks. Internet Measurements Conference, October 2005.

[14] Srinivasan Keshav. A control-theoretic approach to flow control. In SIGCOMM '91: Proceedings of the conference on Communications architecture \& protocols, pages 3-15, New York, NY, USA, 1991. ACM Press.

[15] Mark Davis. A Wireless Traffic Probe for Radio Resource Management and QoS Provisioning in IEEE 802.11 WLANs. ACM Symposium on Modeling, Analysis and Simulation of Wireless and Mobile Systems (MSWiM'04), October 2004.

[16] Henrik Lundgren, Erik Nordstrm, and Christian Tschudin. Coping with communication gray zones in ieee $802.11 \mathrm{~b}$ based ad hoc networks. In 5th ACM international workshop on Wireless mobile multimedia (WoWMoM 2002, pages 49-55. ACM Press, 2002. Available at http://www.it.uu.se/research/group/core/publications/ lundwowmom2002.pdf.

[17] Karol Kowalik, Brian Keegan, and Mark Davis. RARE Resource Aware Routing for mEsh. IEEE International Conference on Communications, June 2007.

[18] Hakim Badis and Khaldoun Al Agha. QOLSR, QoS routing for Ad Hoc Wireless Networks Using OLSR. European Transactions on Telecommunications, 15(4), 2005.

[19] Hakim Badis and Khaldoun Al Agha. Distributed Algorithm for Multiple-metric Link State QoS Routing Problem. IEEE MWCN2003, October 2003.

[20] Francisco J. Ros. UM-OLSR. available at http://masimum.dif. um.es/? Software: UM-OLSR.

[21] Eddie Kohler, Robert Morris, Benjie Chen, John Jannotti, and M. Frans Kaashoek. The Click modular router. ACM Transactions on Computer Systems, 4(18), November 2000.

[22] Xiuchao Wu and A. L. Ananda. Link Characteristics Estimation For IEEE 802.11 DCF Based WLAN. 29th Annual IEEE Conference on Local Computer Networks (LCNO4), November 2004.

[23] F. Schmidt-Eisenlohr, J. Letamendia-Murua, M. Torrent-Moreno, and H. Hartenstein. Bug Fixes on the IEEE 802.11 DCF module of the Network Simulator ns-2.28. Technical Report TR-2006-1, Department of Computer Science, University of Karlsruhe, January 2006. 